\title{
COMMENT
}

\section{Statistical basis and outputs of stable isotope mixing models: Comment on Fry (2013)}

\author{
Brice X. Semmens ${ }^{1, *}$, Eric J. Ward ${ }^{2}$, Andrew C. Parnell ${ }^{3}$, Donald L. Phillips ${ }^{4}$, \\ Stuart Bearhop ${ }^{5}$, Richard Inger ${ }^{6}$, Andrew Jackson ${ }^{7}$, Jonathan W. Moore ${ }^{8}$ \\ ${ }^{1}$ Scripps Institution of Oceanography, University of California San Diego, 9500 Gilman Drive, La Jolla, \\ California 92039-0202, USA \\ ${ }^{2}$ Conservation Biology Division, Northwest Fisheries Science Center, National Marine Fisheries Service, National Oceanic \\ and Atmospheric Administration, 2725 Montlake Blvd. East, Seattle, Washington 98112, USA \\ ${ }^{3}$ School of Mathematical Sciences (Statistics), Complex and Adaptive Systems Laboratory, University College Dublin, Ireland \\ ${ }^{4}$ U.S. Environmental Protection Agency, National Health and Environmental Effects Research Laboratory, \\ Western Ecology Division, 200 SW 35th St., Corvallis, Oregon 97333, USA \\ ${ }^{5}$ Centre for Ecology and Conservation, School of Biosciences, University of Exeter, Exeter EX4 4SB, UK \\ ${ }^{6}$ Environment and Sustainability Institute, School of Biosciences, University of Exeter, Exeter EX4 4SB, UK \\ ${ }^{7}$ Department of Zoology, School of Natural Sciences, and Centre for Biodiversity Research, Trinity College Dublin, Dublin 2, \\ Ireland \\ ${ }^{8}$ Earth to Ocean Research Group, Simon Fraser University, 8888 University Drive, Burnaby, British Columbia V5A 1S6, Canada
}

\begin{abstract}
Fry (2013; Mar Ecol Prog Ser 472:1-13) reviewed approaches to solving underdetermined stable isotope mixing systems, and presented a novel approach based on graphical summaries. He inaccurately characterized the statistics and interpretation of outputs from IsoSource and more recent Bayesian mixing model tools (e.g. SIAR, MixSIR), however, and as an alternative promoted an approach — not based on likelihood methods — that uses graphing and 2 new metrics for tracking source contributions to a mixture. Fry's approach does not provide statistical probability densities associated with source contribution parameter estimates, has little applicability to complex mixing systems such as hierarchical models, and relies on the subjective interpretation of graphing products. We clarify the analytic theory underlying common mixing model approaches and provide an analysis of the 4-source, 2-tracer underdetermined mixing system example in Fry (2013), using both a Bayesian mixing model and Fry's graphical analysis and summary metrics. We demonstrate that properly interpreted Bayesian approaches yield distributions of parameter estimates that can reflect multi-modality, covariance and parameter uncertainty.
\end{abstract}

KEY WORDS: Bayesian mixing model $\cdot$ SIAR $\cdot$ MixSIR $\cdot$ IsoSource

Resale or republication not permitted without written consent of the publisher

\section{Introduction}

The science of stable isotope mixing models has tended towards the development of modeling products (e.g. IsoSource, SIAR, MixSIR) where methodological advances or syntheses are published in tandem with software packages (Phillips \& Gregg 2003, Moore \& Semmens 2008, Parnell et al. 2010). While statistical methods for isotopic mixing models have advanced rapidly, this approach has also afforded an easy avenue toward the application of analytic techniques without a clear understanding of the mechanistic underpinnings. A review by Fry (2013) incorrectly characterized the outputs of existing mixing model tools and promoted alternative methods that are subjective in interpretation and not rooted in a likelihood paradigm (whether maximum likelihood or Bayesian). We clarify the statistical basis for, and 
interpretation of, existing mixing model tools such as IsoSource and SIAR. For illustration, we build on Fry's example of a 4-source, 2-tracer square mixing system to show that existing Bayesian mixing model methods provide a better interpretation of uncertainty in the solution space than Fry's graphical approach and summary metrics.

\section{Statistical basis of mixing models}

Fry (2013) first focused on reporting the outputs of IsoSource and SIAR, stating that both produce point estimates of source contributions to a mixture along with uncertainty intervals based on statistical subsampling. Fry's criticism of these outputs was that sampling frequency does not equate with probability. However, the output frequency of posterior sampling resulting from Bayesian mixing models is precisely related to probability. A plot of the posterior sampling density from a Bayesian model run provides an explicit assessment of the relative likelihood of the entire compositional parameter space (which may or may not feature multiple or flat peaks; Fig. 1). Although IsoSource is based on an empirical rather than a statistical (likelihood-based) approach, IsoSource also produces histograms of source proportions that represent parameter uncertainty due to the underdetermined nature of the system. Unlike

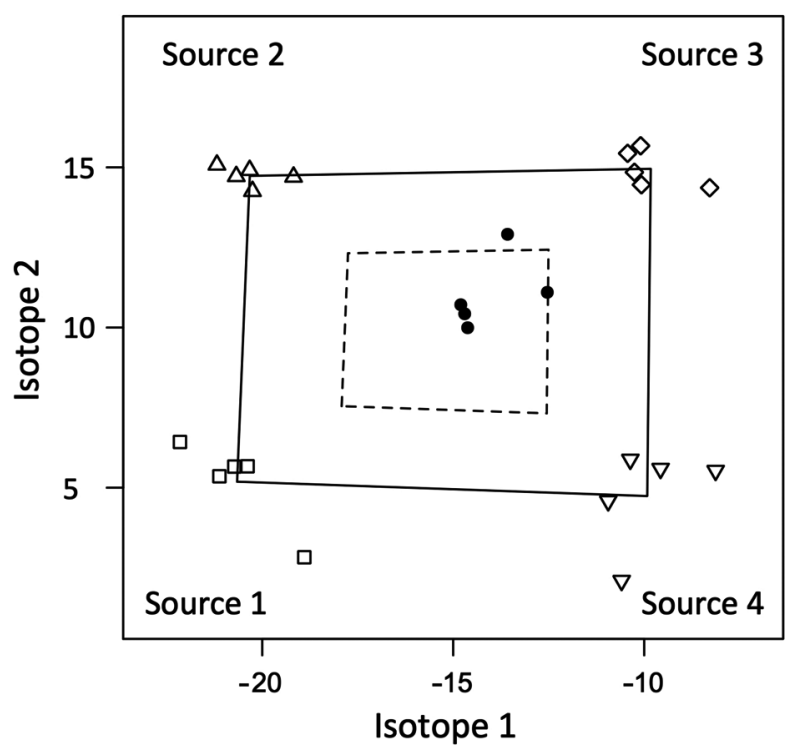

Fig. 1. Simulated source and mixture data, including a source polygon based on source data means and a 'danger zone' polygon halfway between the source polygon and the centroid $\left(\Sigma_{\mathrm{MIN}}<50 \%\right.$, where $\Sigma_{\mathrm{MIN}}$ is the sum of minimum source contributions calculated by IsoSource or SIAR)
Bayesian tools (i.e. SIAR or MixSIR), IsoSource uses only source and mixture isotopic means and does not propagate uncertainty due to isotopic (or trophic enrichment factor) variability. Because Fry (2013) did not consider the philosophical differences among the 3 modeling approaches (IsoSource, Bayesian, and graphical mixing models), we address them briefly below.

Fry (2013) referred to both IsoSource and SIAR as frequentist in their outputs, but neither method is based on frequentist statistics. In the case of IsoSource, the modeling approach relies on iteratively identifying all sets of proportional source contributions that match the isotope signatures of the mixture, based on a defined tolerance. Unlike frequentist or Bayesian methods, IsoSource does not assess the probability of the mixture data given proportional source contribution values. While a frequentist approach assumes a fixed hypothesis (that is without probability), IsoSource focuses on identifying the range and frequency of source proportion hypotheses that solve the mixing model given the specified tolerance. Fry incorrectly asserted that all source contributions represented in the IsoSource output are equally likely. Within the unique sets of proportions identified by IsoSource, a specific source contribution (say, 30\% for Source 1) may be represented many times, while another (say, 90\% for Source 1) may be represented very infrequently. The frequency of occurrence of a particular source contribution thus characterizes the number of unique solutions associated with that contribution relative to all other contribution values. Because of this, Phillips \& Gregg (2003) emphasized that the outputs of IsoSource should be characterized in terms of the distribution of the solutions, not just of a single value such as the mean or most likely value.

Since Moore \& Semmens (2008) introduced Bayesian mixing models, most analytic advances to mixing model methods have been rooted in Bayesian statistics. Frequentist analyses treat the parameters $(\theta)$ as fixed and the data as random; Bayesian methods assume the opposite - that the parameters are unknown but the data are fixed - and describe uncertainty in the parameters probabilistically based on observed data, $\mathrm{P}(\theta \mid$ data $)$. Because most mixing model studies aim to probabilistically characterize the diet contributions of sources to a mixture based on observed data, rather than to test specific null hypotheses (e.g. 'all source contributions are equal'), a Bayesian approach is prudent (for further advantages of Bayesian methods, see Moore \& Semmens 2008, Parnell et al. 2010, Ward et al. 2010). 
To evaluate the posterior likelihood of source contributions, Bayesian modeling approaches such as SIAR make use of Markov Chain Monte Carlo (MCMC) sampling methods. Fry (2013) incorrectly stated that MCMC techniques perform statistical subsampling of the range of feasible solutions. While this description is an accurate characterization of IsoSource, Bayesian methods such as MCMC approximate the entire posterior distribution of model parameters. A posterior density curve thus provides an explicit estimate of the relative likelihood for all parameter values. For underdetermined mixing models, Fry pointed out that likelihood densities can be multimodal, and that credible intervals (e.g. 95\% CIs) for such estimates can be misleading. We agree, and strongly advocate a graphical representation of the entire posterior distribution of source proportion parameters (Moore \& Semmens 2008); this is a routine component of reporting Bayesian analyses (Chen \& Shao 1998). Multi-modal posterior distributions arise when multiple disparate compositional parameter sets have relatively high likelihood. In such cases, multi-modality may be reduced by: (1) incorporating more consumer data; (2) including prior information (Moore \& Semmens 2008, Ward et al. 2010); (3) changing source geometry by re-grouping sources (Phillips et al. 2005, Ward et al. 2011); (4) including residual error (Parnell et al. 2010). However, there are also cases when isotope data from consumers are simply not informative with respect to estimating source contributions. While Fry criticized credible intervals in these cases as misleading, we think that they represent an accurate reflection of uncertainty and should not be used to justify the abandonment of likelihood based methods (whether frequentist or Bayesian). Finally, while Fry promoted post hoc approaches to the constraint of underdetermined mixing model solutions based on additional information (e.g. gut content data), we note that Bayesian methods provide a concise framework for the direct integration of prior information into the mixing model analysis (Moore \& Semmens 2008).

\section{Graphical vs. Bayesian analysis of underdetermined mixing systems}

The graphical analysis of mixing models proposed by Fry (2013) has no basis in a likelihood framework (whether frequentist or Bayesian), ignores uncertainty in both source and mixture data, and does not provide probabilistic estimates of source contributions. Additionally, it is unclear how the approach might be extended to systems with more than 2 tracers, given the reliance on interpretation through visualization. To use the graphical approach advocated, one must reduce source data to a single mean point, and then construct a polygon chart (our Fig. 1). Within this polygon, Fry proposed the construction of a secondary 'danger zone' polygon that defines halfway points between the exterior polygon sidewalls and the centroid. Fry reasoned that mixture data points falling within this danger zone are dominated by the assumed centroid contributions and are little constrained by the measured data. Such a plotting exercise does not make it possible to draw concise conclusions about source contributions to a mixture. Like Fry, we advocate plotting isotope data in the context of mixing model geometry to evaluate the utility of a mixing model approach. However, we see no advantage in relating mixture data to an arbitrary 'danger zone' polygon over the graphical representation of posterior source contribution parameters from Bayesian mixing model approaches.

Bayesian methods are powerful for addressing questions of cause and consequence (e.g. Giroux et al. 2012), where the key objective is to identify differences in foraging patterns among different populations, demographic groups, or individuals. Such approaches do not depend on precise measures of consumption, because they focus on inference of differences among groups. By quantifying the variation at different levels of social organization in the consumer population, Bayesian mixing models can decompose the trophic niche of a population into associated levels of population structure (Semmens et al. 2009). In contrast, the interpretation of niche space in a multi-level mixture population using graphical analysis lacks an objective basis.

Fry (2013) proposed 2 mathematical summary calculations that provide metrics for the strength of mixing model solutions, based on the outputs of IsoSource and SIAR: $\Sigma_{\mathrm{MIN}}$, the sum of minimum source contributions calculated by IsoSource or SIAR, and \% resolved, the difference between the minimum and maximum source contributions, subtracted from 100. We think that the summary metrics proposed suffer the same limitations as parameter credible intervals, namely that they will fail to accurately characterize the likelihood distribution for source contribution parameters when such distributions are multimodal (e.g. Fig. 2). Both of Fry's metrics provide mathematical descriptors of the range of mixing solutions, but neither provides an explicit assessment of the uncertainty in the estimates of proportional source contributions to the mixture. 


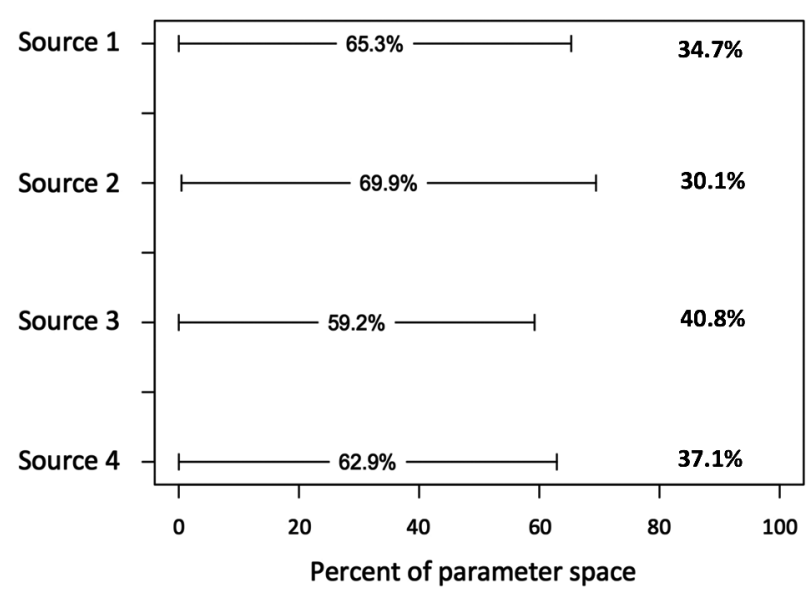

Fig. 2. Resolved metrics (\%) for each source based on the proportional source contribution parameter posterior densities generated by a fully Bayesian mixing model. Bars: parameter ranges for each source contribution parameter; bold: resolved values ( 1 - range, \%)

\section{Contrasting Bayesian and graphical approaches to mixing models: an example}

To facilitate a discussion of the relative merits of Bayesian versus graphical mixing models, we expand on Fry's (2013) 4-source, 2-tracer example (his Fig. 1A). Specifically, we maintain the geometry of the example, but add variability into the data (for all sources and the mixture, $\mathrm{n}=5$, variance $=1.3$; our Fig. 1). The addition of variability to the example more accurately reflects the type of data that ecological mixing model studies rely on. For instance, this sample size and variability matches that of the mangrove source data from the case study Fry addressed (Kon et al. 2009). Next, we construct a graph of the source and danger area polygons $\left(\Sigma_{\mathrm{MIN}}<50 \%\right.$; Fig. 1) and a graph of the \% resolved metric advocated by Fry (2013; our Fig. 2). Finally, we build and apply a simple fully Bayesian mixing model (Ward et al. 2010) and generate posterior distributions for the proportional contribution terms (Figs. $3 \& 4$ ). The R code used for data simulation and all analyses is provided in the Supplement at www.int-res.com/articles/ suppl/m490p285_supp/.

The posterior distributions for the proportions in our example reflect multi-modality (Fig. 3). Given this multi-modality, a researcher would be remiss in reporting the posterior distributions based solely on summary statistics such as means or medians with associated credible intervals. Bayesian methods are at their most powerful when models yield complex posterior likelihoods, since plots of posterior surfaces accurately characterize the full breadth of this complexity. In contrast, the \% resolved metric proposed by Fry (2013; our Fig. 2) suffers the same limitation that Bayesian credible interval summary statistics have when reported without a plot of posterior densities - they only characterize the range of solutions without identifying modality in the parameter likelihoods and provide little insight into the covariance of the parameter solutions.

Fig. 4 indicates that the posterior distributions of the source contributions are highly correlated. This is a direct result of the geometry of the system and

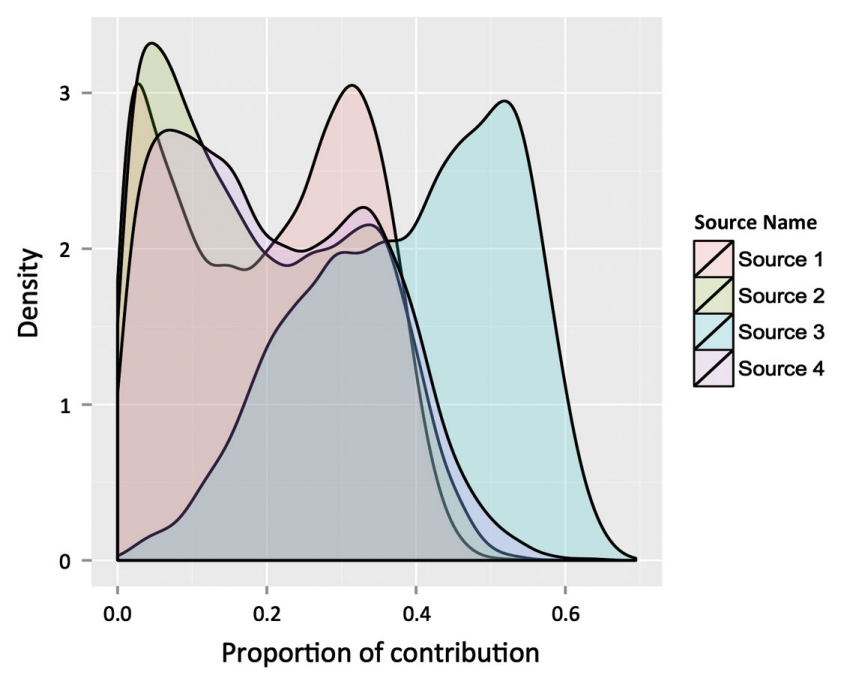

Fig. 3. Posterior densities of proportional source contribution parameter generated by a fully Bayesian mixing model

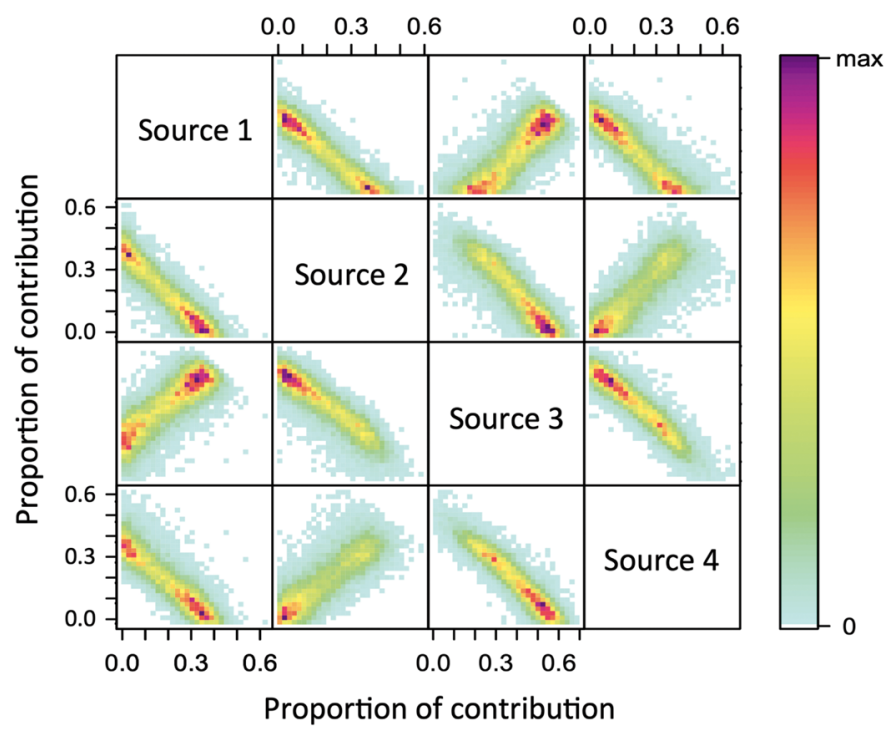

Fig. 4. Draftsman's plot of posterior source contributions, showing interrelations between the posterior parameter densities 
leads to unavoidable uncertainty in the individual (marginal) source contribution estimates (shown in Fig. 3). If these correlations are not reported, it may lead to mis-interpretation of the model output, e.g. when reporting (from Fig. 3) that the proportions for both Source 1 and Source 2 might be around 0.3, when in fact if the proportion from Source 1 is large, the proportion from Source 2 is necessarily small. We see these correlation plots as being vital to the evaluation of model behavior.

Finally, the interpretation of Fry's (2013) graphical approach presents a challenge when realistic variability occurs in isotope data. In our example, some of the mixture data fall outside the danger zone polygon, due exclusively to random error. In such a situation, a researcher might optimistically conclude that a portion of the mixture population supports mixing model interpretation based mostly ( $>50 \%$ ) on measurement rather than assumption. On the other hand, the majority of the mixture data fall within the danger zone, suggesting (pessimistically) that the geometry of the source data leads principally to interpretation based on assumption (using Fry's terminology). Bayesian mixing models avoid this subjectivity by affording a synthesis of the source and mixture data in a model framework that integrates data variability in the generation of posterior parameter estimates. These posterior parameter estimates thus provide a clear picture of the extent of uncertainty in source contributions to the mixture population.

\section{Concluding remarks}

Plotting stable isotope data is a necessary first step in any mixing model; however, such plotting exercises by themselves do not adequately characterize uncertainty in parameter estimates. Fry (2013) highlighted the importance of such plots, and provided guidance for interpreting these plots in the context of isotope geometry. Fry advocated applying his graphical techniques and summary statistics in concert with existing mixing model outputs. Implicit in such a dual approach is the assumption that the outputs of existing mixing models do not provide actual statistical probability densities associated with source contribution parameter estimates. This assumption is accurate for IsoSource, but not for the more recent class of Bayesian mixing models. When properly applied, Bayesian mixing model tools yield complete posterior probability densities for source contribution parameters that encapsulate both variability in observed data and uncertainty due to isotope geometry.

\section{LITERATURE CITED}

Chen MH, Shao QM (1998) Monte Carlo estimation of Bayesian credible and HPD intervals. J Comput Graph Statist 8:69-92

> Fry B (2013) Alternative approaches for solving underdetermined isotope mixing problems. Mar Ecol Prog Ser 472: $1-13$

Giroux MA, Berteau D, Lecomte N, Gauthier G, Szor G, Bêty J (2012) Benefiting from a migratory prey: spatio-temporal patterns in allochthonous subsidization of an arctic predator. J Anim Ecol 81:533-542

Kon K, Kawakubo N, Aoki J, Tongnunui P, Hayashizaki K, Kurokura H (2009) Effect of shrimp farming organic waste on food availability for deposit feeder crabs in a mangrove estuary, based on stable isotope analysis. Fish Sci 75:715-722

Moore JW, Semmens BX (2008) Incorporating uncertainty and prior information into stable isotope mixing models. Ecol Lett 11:470-480

> Parnell AC, Inger R, Bearhop S, Jackson AL (2010) Source partitioning using stable isotopes: coping with too much variation. PLoS ONE 5:e9672

> Phillips DL, Gregg JW (2003) Source partitioning using stable isotopes: coping with too many sources. Oecologia 136:261-269

Phillips DL, Newsome SD, Gregg JW (2005) Combining sources in stable isotope mixing models: alternative methods. Oecologia 144:520-527

Semmens BX, Ward EJ, Moore JW, Darimont CT (2009) Quantifying inter- and intra-population niche variability using hierarchical Bayesian stable isotope mixing models. PLoS ONE 4:e6187

- Ward EJ, Semmens BX, Schindler DE (2010) Including source uncertainty and prior information in the analysis of stable isotope mixing models. Environ Sci Technol 44: 4645-4650

- Ward EJ, Semmens BX, Phillips DL, Moore JW, Bouwes N (2011) A quantitative approach to combine sources in stable isotope mixing models. Ecosphere 2:art19

Submitted: April 10, 2013; Accepted: August 26, 2013

Proofs received from author(s): September 9, 2013 